\title{
ENHANCED EIGENSTRUCTURE ASSIGNMENT FOR AEROELASTIC CONTROL APPLICATION
}

\author{
M. Merkel, M. H. Gojny and U. B. Carl \\ Technical University of Hamburg-Harburg \\ Department of Aircraft Systems Engineering \\ 21071 Hamburg, Germany
}

\begin{abstract}
Regarding the regulation of weakly damped aeroelastic structures by means of the primary flight controls (PFC), the present contribution focusses on a sophisticated robust controller design method, to achieve the required functionality. Generally, the outlined approach is premised on eigenstructure assignment. Enhancements promise effective integration of miscellaneous potentially competing objective specifications either in time or frequency domain as well as parametric requirements. Due to the infinitive set of flight conditions and fuel configurations the underlying aeroservoelastic plant consists of a linear multivariable multi-model system. Technical feasibility, parametric and dynamic uncertainties as well as architectural constraints, e.g. the number of available sensors lead to a robust output feedback controller. Principally the proposed method is well known from flight control design and modal decoupling. But in contrast, the constrained simultaneous robust eigenvalue stabilisation represents the primary objective at the current application. Since the intrinsic controller calculation is implemented as multiobjective optimisation task, proper optimisation criteria are defined, which incorporate the aeroservoelastic performance specifications into the design procedure. The suitability of the resulting loworder static output controller is verified by real time simulation for a completely normal and degraded configuration covering a real PFC actuation system hardware-in-theloop.
\end{abstract}

\section{KEYWORDS}

Flexible aircraft, aeroservoelasticity, structural vibrations, flutter margin, damping augmentation, robust controller design, eigenstructure assignment, hardware-in-the-loop simulation

\section{INTRODUCTION}

Aeroelastic control can be more challenging than conventional controlled structures problem, in that the dynamics of the system change dramatically with the flight conditions. It holds the promise of significant improvements in performance: reducing the ambient vibration level, increasing the maneuver responsiveness and stabilising an otherwise unstable system (Dowell 1995).
Considering a commercial aircraft, aerodynamic forces and moments entail a substantial deformation of the elastic structures: fuselage, tailplane and wing. Each incremental change of the structural shape yields a new aerodynamic state which causes aeroelastic interaction. Due to the increasing size of transport aircraft, high aspect ratio and new materials like composites, the spectral gap between flightmechanical motion and structural modes decreases continuously (Schuler 1998, Lind and Brenner 1999). Hence, dynamically coupled modes of the flexible aircraft arise, which can be excited by gust loads as well as maneuvers and flight mechanical stability augmentation functions (FIG 1). Neglecting supplementary measures, these vibratory modes are usually characterised by a weak damping ratio. The resultant mechanical vibrations reduce the fatigue life of the structure, impair ride comfort and structural loads and may lead, under worst conditions, to a complete loss of the aircraft's controllability. In order to solve this problem for light weight structures, much effort has been spent in the last decade aiming at an additional functionality of the primary flight control surfaces, to counteract gust and maneuver induced aeroelastic vibrations. With respect to the changing flight operation conditions, e.g. speed and fuel mass, the physical parameters of the aeroservoelastic plant vary considerably.

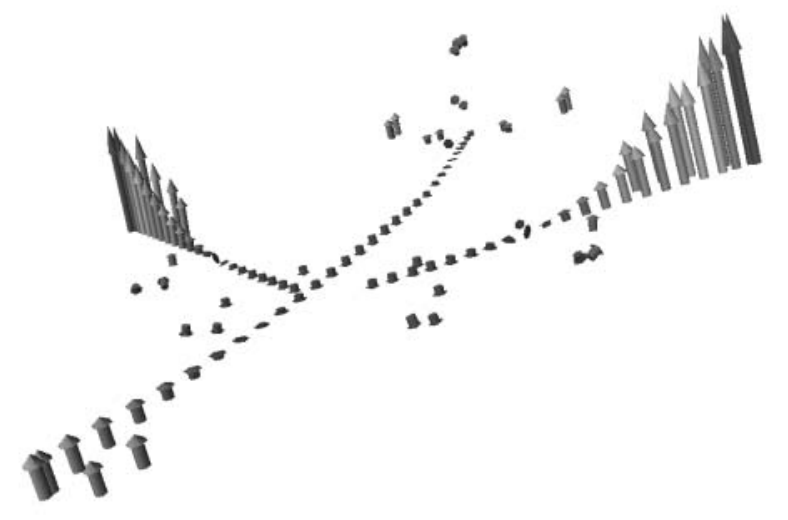

FIG 1: Aeroelastic deformation 
Additionally, nonlinearities and neglected dynamics increase the level of uncertainty. Due to the presence of parametric and dynamic uncertainties, the active modal damping augmentation must be distinguished by strong robustness. Issues of system implementation, e.g. limited number of sensors, discrete-time realisation, etc. entail remarkable restrictions to the feedback concept. Meeting this challenge, a variety of different methodologies have already been proposed for civil aircraft applications, e.g. classical Nyquist method plus filtering (Seyffarth 1993), mixed control (Kubica 1995), i.e. successively modal flight plus LQ optimal vibration control design, $\mathcal{H}_{\infty}$ Loop Shaping control (Schuler 1998), tunable model-based LQ optimal control (Chiappa et al. 1998), convex synthesis (Boyd and Barrat 1991, Dardenne 1998), LQG and $\mu$-synthesis (Hanel 2001, Mukhopadhyay et al. 2001) and application of genetic algorithms (Wu 1999). Since current vibration control is dominated by norm-weighted optimal controller design methods, engineers frequently complain the lack of physical interpretation and compatibility with the real aircraft system environment, i.e. implementable time constants, calculation effort of dynamic controllers etc.. Consequently, a robust low-order static output feedback simultaneously stabilising the entire set of plant representatives is expected to grant an effort-performance ratio with good prospects (Kubica 1995). Indeed, the order of the output controller need not mandatorily be associated with the number of objective modes to be controlled.

Since modal decoupling is a lower level consideration in this context of active modal control, the problem of eigenspace specification - (Kubica and Livet 1994) - has been transformed to a simultaneous robust stabilisation task. Generally, eigenstructure assignment conserves advantageous properties: control engineers are familiar with the design procedure, conservation of physical interpretation, illustrative performance specifications, analytical synthesis approach, etc.. Synthesising eigenstructure assignment, Roppenecker's formula (Roppenecker 1990) — and principles from robust parameter space design method (Ackermann et al. 1993) lead to enhanced eigenstructure assignment for simultaneous $\Gamma$ stabilisation. This modus operandi even covers restrictions due to the system architecture, system degradation as well as implementation issues.

This contribution presents the definition of the modified design procedure and application to a generic aeroservoelastic multiple-input multiple-output system (MIMO system), exemplarily. The paper is organised as follows: Chapter 2 discusses the proposed controller design method in brief and focusses on different types of performance specifications. The conformation of the aeroservoelastic plant is presented in chapter 3. Additionally the associated basic properties are outlined. Beyond, the application of this method to the aeroservoelastic plant as well as the verification of the closed loop system dynamics are outlined in chapter 4. Concluding remarks finalise this paper.

\section{ENHANCED EIGENSTRUCTURE ASSIGNMENT}

Incorporating the basic state-space approach and the idea of uncertainty and robustness in terms of Ackermann (Ackermann et al. 1993) into a modern MIMO controller design method bridges the gap between methodologies for controller development in the time and frequency domain. Transparently, both classes of system requirements are integrated within one design procedure even for large scale systems. The optimisation core rests upon a constrained nonlinear programming, denoted as sequential quadratic programming (SQP). This chapter presents a brief description of the proposed enhanced eigenstructure assignment method robust output feedback design.

Approach. Assuming the uncertain plant is denoted by a linear time-invariant multi-model-system with $l$ statespace representatives

$$
\begin{aligned}
\dot{\boldsymbol{x}}(t) & =\boldsymbol{A}(\boldsymbol{q}) \mathbf{x}(t)+\boldsymbol{B}(\boldsymbol{q}) \mathbf{u}(t) \\
\mathbf{y}(t) & =\boldsymbol{C}(\boldsymbol{q}) \mathbf{x}(t)+\boldsymbol{D}(\boldsymbol{q}) \mathbf{u}(t), \quad \boldsymbol{x}(0)=\mathbf{x}_{0},
\end{aligned}
$$

Each representative is marked by $n$ states, $p$ inputs and $m$ outputs. Uncertainties are assumed to be concentrated within the bounded uncertainty vector $\boldsymbol{q}$ (Gojny 2001). Fundamentally, this approach rests upon Roppenecker's formula,

$$
\text { (2) } \boldsymbol{R}=\boldsymbol{P} \boldsymbol{W}^{-1}
$$

wherein the static controller gain matrix $\boldsymbol{R}$ results from the system description of the plant, system matrix $\boldsymbol{A}$ and input matrix $\boldsymbol{B}$, the desired closed loop eigenvalues $\lambda_{R i}, i=$ $1 \ldots n$

$$
\boldsymbol{W}=\left[\begin{array}{c}
\left(\boldsymbol{A}-\lambda_{R 1} \boldsymbol{I}\right)^{-1} \boldsymbol{B} \boldsymbol{p}_{1} \\
\vdots \\
\left(\boldsymbol{A}-\lambda_{R n} \boldsymbol{I}\right)^{-1} \boldsymbol{B} \boldsymbol{p}_{n}
\end{array}\right]^{\mathrm{T}}
$$

and the assigned parameter vectors

$$
\boldsymbol{P}=\left[\begin{array}{lll}
\boldsymbol{p}_{1} & \cdots & \boldsymbol{p}_{n}
\end{array}\right] .
$$

Since the eigenvalue specifications of the closed loop system are completely determined by the desired root locations $\lambda_{R i}$, the parameter vectors $\boldsymbol{p}_{i}$ represent the remaining degrees of freedom to influence the MIMO plant regarding further objectives. Due to their direct linkage to the closed loop eigenvectors $\mathbf{v}_{R i}$

$$
\boldsymbol{p}_{i}=\boldsymbol{R} \mathbf{v}_{R i}, \quad i=1 \ldots n
$$

these parameter vectors $\boldsymbol{p}_{i}$ are often used to assign a predeterminated eigenspace. Commonly, this procedure is denoted as generalised eigenstructure assignment (She 1992, Livet 1995), whereas the assignable eigenspace is limited by the number of inputs $p$ only. 


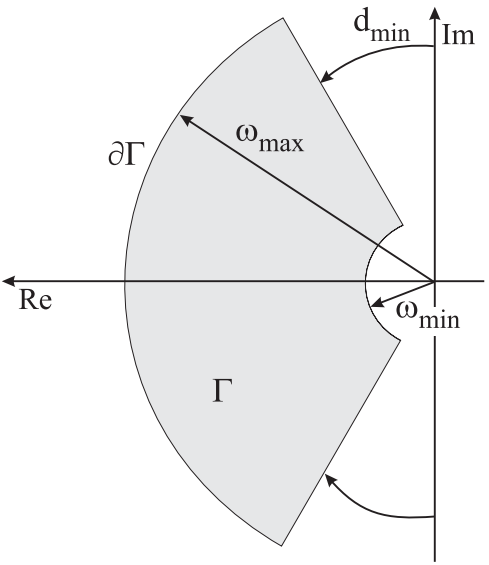

FIG 2: Region of eigenvalues $\Gamma$

Performance specifications. Considering state-space models, performance requirements can easily be transformed to the closed loop eigenvalue locations in the complex plane. The active vibration control function grants all eigenvalues of the current representative to be always arranged within the specified region $\Gamma$, which is bounded by a minimum damping $d_{\min }$, a maximum rise time $\omega_{\min }=1 / T_{\max }$, and a maximum bandwidth $\omega_{\max }$ (FIG 2). Ensuring the specified system dynamics are met in closed loop by all considered plants is qualified by the robustness requirement.

In addition to the dynamic performance specification, further requirements are considered in terms of norm-based criteria as e.g. the regulation of small controller gains, by its weighted absolute value, architectural constraints, which enables to select particular feedback variables to obtain an output feedback and power-optimised state or input-output transitions just like the Riccatti performance index (Roppenecker 1990).

The goal of the design procedure is to find a controller matrix $\boldsymbol{R}$, which simultaneously moves the preselected eigenvalues of the entire set of representatives into the desired pole region $\Gamma$ and minimises the set of performance indexes.

Design variables and optimisation. Contrary to eigenvalues the demand for eigenvectors and respectively the parameter vectors can not be clearly determinated, which formerly aggravated eigenstructure assignment in vibration control application (Kubica and Livet 1994). The subsequent approach remedies that nuisance limiting only the absolute value of the parameter vector, i.e. its length. Applying (2) to the scaled parameter vectors

$$
\tilde{\boldsymbol{p}}_{i}=c_{i} \boldsymbol{p}_{i}, \quad c_{i} \in \mathbb{R} \backslash 0, i=1, \ldots, n,
$$

yields the resulting controller matrix $\tilde{\boldsymbol{R}}$, which obviously is equal to the controller matrix $\boldsymbol{R}$ (3) (Roppenecker 1990). Hence, restricting the length of the parameter vectors does not restrict the eigenspace of the closed loop system, but is necessary for a well conditioned optimisation problem.

The optimisation is carried out by standard functions from e.g. Matlab's Optimisation Toolbox. Although simultaneous performance vector optimisation is standard implementation, transforming the design task to an less costly optimisation problem, in which the constrained minimum of a scalar function is sought for, frequently suffices regarding the current design task (Föllinger 1994, Roppenecker 1990). Hence, the total performance index results as a linear weighted combination of subindexes.

Eigenvalues and parameter vectors are chosen as design variables and arranged as vector $\boldsymbol{L}$, its length governs the search space of the optimisation. The constrains are defined by a set of lower and upper bounds $L^{-}$and $L^{+}$referring to each design variable $L$. Assuming plants in state space form, the eigenvalue representation deviates from the common description

$$
\lambda_{1,2}=-\delta_{e} \pm \mathrm{j} \omega_{e}
$$

which usually implies a damping coefficient $d<1$. To enlarge the edge of representable pole configurations for optimisation purposes requires a strict real representation even covering, e.g. $d>1$

$$
\lambda_{1,2}=-\omega d \pm \omega \sqrt{d^{2}-1} .
$$

The corresponding parameter vector are assigned complex conjugate as well

$$
p_{1,2}=\left[x_{1} \ldots x_{p}\right]^{\mathrm{T}} \pm \mathrm{j}\left[y_{1} \ldots y_{p}\right]^{\mathrm{T}} .
$$

Thus the eigenvalues and parameter vectors form $\boldsymbol{L}$ of length $\operatorname{dim}(\boldsymbol{L})=n(p+1)$,

$$
\begin{aligned}
& \boldsymbol{L}=\left[\begin{array}{llllll}
\omega_{1} & \ldots & \omega_{\left(n-\frac{c}{2}\right)} & d_{1} & \ldots & d_{\frac{c}{2}}
\end{array}\right. \\
& \left.\begin{array}{lllllll}
\cdots & x_{1} & \ldots & x_{p \cdot\left(n-\frac{c}{2}\right)} & y_{1} & \ldots & y_{p \cdot \frac{c}{2}}
\end{array}\right],
\end{aligned}
$$

whereas $c$ denotes the number of complex conjugate root loci. The upper and lower bounds of the design parameters are denoted

$$
\begin{aligned}
& \boldsymbol{L}^{+}=\left[\begin{array}{llllll}
\omega_{\max } & \ldots & \omega_{\max } & d_{\max } & \ldots & d_{\max }
\end{array}\right. \\
& \left.\begin{array}{lllllll}
\cdots & 1 & \ldots & 1 & 1 & \ldots & 1
\end{array}\right], \\
& \boldsymbol{L}^{-}=\left[\begin{array}{llllll}
\omega_{\min } & \ldots & \omega_{\min } & d_{\min } & \ldots & d_{\min }
\end{array}\right. \\
& \left.\begin{array}{lllllll}
\ldots & -1 & \ldots & -1 & -1 & \ldots & -1
\end{array}\right],
\end{aligned}
$$

Whereas their first part arises from the eigenvalue bounds $\partial \Gamma$, fundamentally, the parameter vector bounds are set to 1 and -1 respectively (6).

The solver applied to the stated problem rests upon sequential quadratic programming, whereas the optimisation algorithm successively minimises a composed criterion $J$. Each iteration loop consists of: 
1. Setting start values to the design variables $\boldsymbol{L}$ with respect to $\boldsymbol{L}^{+}, \boldsymbol{L}^{-}$or taking over the previous result.

2. Transforming $\boldsymbol{L}$ into the corresponding eigenvalues $\lambda_{R 1}, \ldots, \lambda_{R n}$ and parameter vectors $\boldsymbol{p}_{1}, \ldots, \boldsymbol{p}_{n}$

3. Evaluating (2) for a nominal or critical plant

4. Applying the controller $\boldsymbol{R}$ to the remaining plants and calculating their eigenvalues and parameter vectors

5. Deriving the primary, robust optimisation criteria $J_{R}$ and further criteria.

6. Valuating the total performance index

Performance specifications and constraints. The primary optimisation criteria $J_{R}$ valuates the root loci of the closed loop systems with respect to the robustness specification $\Gamma$ (FIG 2)

$$
J_{R}\left(\lambda_{R i}(\boldsymbol{R}), \Gamma\right)=\sum_{k=1}^{l} \sum_{i=1}^{n} \gamma_{k i}
$$

All eigenvalues are rated according to their distance to the edge of $\Gamma$ (FIG 3). The farer the distance, the higher the penalty according to

$$
\begin{aligned}
& \gamma_{k i}=\left|\omega_{c}-\omega_{k i}\right|+\left|d_{c}-d_{k i}\right| \\
& \omega_{c}=\left\{\begin{array}{rll}
\omega_{k i} & : & \omega_{\min } \leq \omega_{k i} \leq \omega_{\max } \\
\omega_{\min } & : & \omega_{\min }>\omega_{k i} \\
\omega_{\max } & : & \omega_{\max }<\omega_{k i}
\end{array}\right. \\
& d_{c}=\left\{\begin{array}{lll}
d_{k i} & : & d_{\min } \leq d_{k i} \leq d_{\max } \\
d_{\min } & : & d_{\min }>d_{k i} \\
d_{\max } & : & d_{\max }<d_{k i}
\end{array}\right.
\end{aligned}
$$

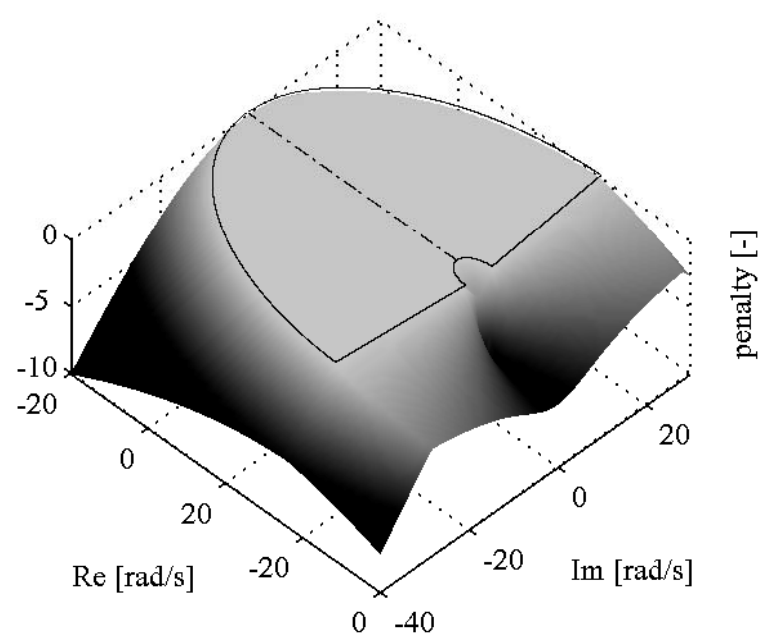

FIG 3: Penalty function
Since eigenvalues of the nominal plant are placed within the desired eigenvalue region as per definition, they do not contribute to $J_{R}$. If a robust stabilising controller exists, the the primary criteria will be equal zero, $J_{R}=0$. Hence all $n \cdot l$ roots are placed within $\Gamma$ simultaneously, either the algorithm terminates or evaluates further criteria. Additionally, according to the small gain theorem small controller gains $r_{j i}$ naturally increase robustness margins of intrinsically stable multi-model systems (Lind and Brenner 1999). In order to consider the absolute values of the resulting static gains coefficients within $\boldsymbol{R}$, the norm criterion

(14) $J_{N}=\sum_{j=1}^{p} \sum_{i=1}^{n} r_{j i}^{2}$.

is stated to influence the total performance index. With regard to architectural constraints (Roppenecker 1990) proposes a structural performance index, which weights feedback coefficients from unavailable states with grave penalties

$$
\begin{array}{ll}
J_{S}=\sum_{j=1}^{p} \sum_{i=1}^{n} g_{j i}^{S} r_{j i}^{2} \\
g_{j i}^{S}>0 & \text { for } \quad r_{j i} \rightarrow 0 \\
g_{j i}^{S}=0 & \text { otherwise. }
\end{array}
$$

Indeed, the assignable space decreases, but often very small gains $r_{j i} \ll 1$ suffice to neglect these feedback signals. Consequently, even output feedback controller requirements are incorporated in transparent manner. Additional criteria are presented in (Roppenecker 1990), moreover the outlined design method allows to customise the proposed criteria, e.g. separated pole region assignment or classical decoupling. The latter can be easily achieved by trimming the parameter vector part of the bounding vectors $\boldsymbol{L}^{+}, \boldsymbol{L}^{-}$plus introduction of a corresponding valuation criterion.

\section{AEROSERVOELASTIC PLANT}

In order to demonstrate the substantial properties of described controller design method the underlying aeroservoelastic plant is condensed to a transparent analytic format. Since the modelling technique in itself is not subject of the current contribution, significant assumptions and prerequisites are collocated in (Gojny 2001). The subsequent chapter mainly focusses on qualitative description, maintaining an illustrative systematic perception.

\subsection{Aeroelastic Model}

In practice, aeroservoelastic modelling of distributed parameter systems entails plants of extensive complexity from a control theory point of view. Merging aerodynamics, elastomechanics, servohydraulics and control theory approaches leads to very complicated interactions, which usually conditions a quite detailed model representative. To perform any analytical approach, a lumped parameter estimation is advantageous. Considering this investigation, the proposed approach is applied to an elementary model exhibiting some dominant properties, which can be seen 


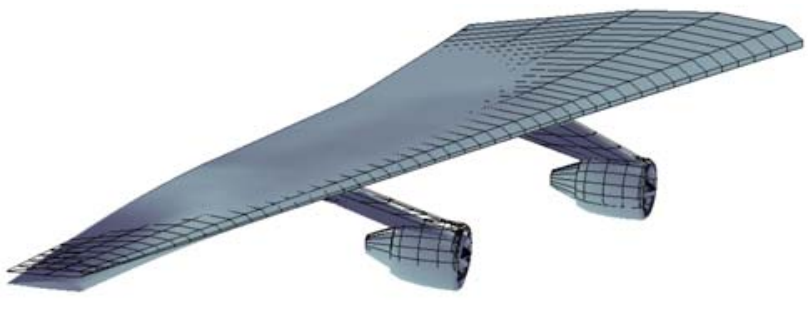

FIG 4: Half span finite element wing model

at current flexible aircraft configurations in vaster shapes.

The aeroelastic subsystem under consideration comprises a half span finite element wing model (FIG 5) which contains the wing box, the inner and outer engine pylon. The mass distribution among the element nodes results from an estimation of the continuously distributed structural mass $\boldsymbol{M}_{S}$, the concentrated masses of the engines $\boldsymbol{M}_{E}$, landing gear and systems. Additionally, arbitrary fueling configurations are estimated by means of scalable fuel tanks $\rho_{i} \in[0,1], i=1 \ldots 3$ (FIG 6)

$$
\boldsymbol{M}=\boldsymbol{M}_{S}+2 \boldsymbol{M}_{E}+\sum_{i} \rho_{i} \boldsymbol{M}_{\text {fuel }}+\cdots
$$

Aerodynamic forces are applied by means of a quasistationary approach. The aerodynamic coefficients result from a spanwise polynomial interpolation of measured pressure distributions (MacKinnon and Stollery 1993). Thus, supposing a generalised surface deflection $\delta$, sections without controls naturally obtain $c_{l \delta}=c_{m \delta}=0$ (Gojny 2001). The pressure distribution for each airfoil section is influenced by the local angle of attack $\alpha$, possibly, the control surface deflection $\delta$ and the dynamic pressure $q=0.5 \rho v_{\mathrm{TAS}}^{2}$ with the true airspeed $v_{\mathrm{TAS}}$. Assuming the wingspan coordinate $y_{l}$ yields

$$
\alpha\left(y_{l}, t\right)=\alpha_{0}(t)+\theta\left(y_{l}, t\right)-\frac{\dot{z}\left(y_{l}, t\right)}{v_{\mathrm{TAS}}} .
$$

Altogether, the entire aeroelastic system behaviour is governed by an ordinary second order, linear differential ma-

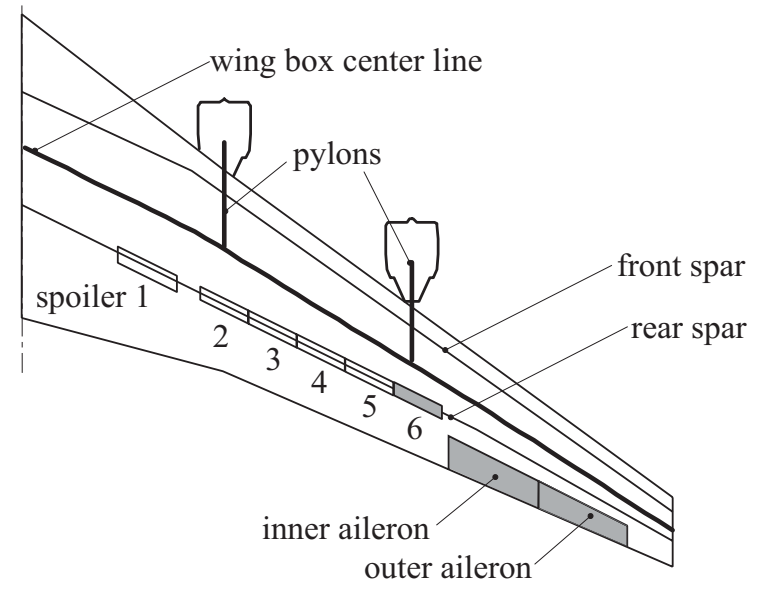

FIG 5: Wing geometry

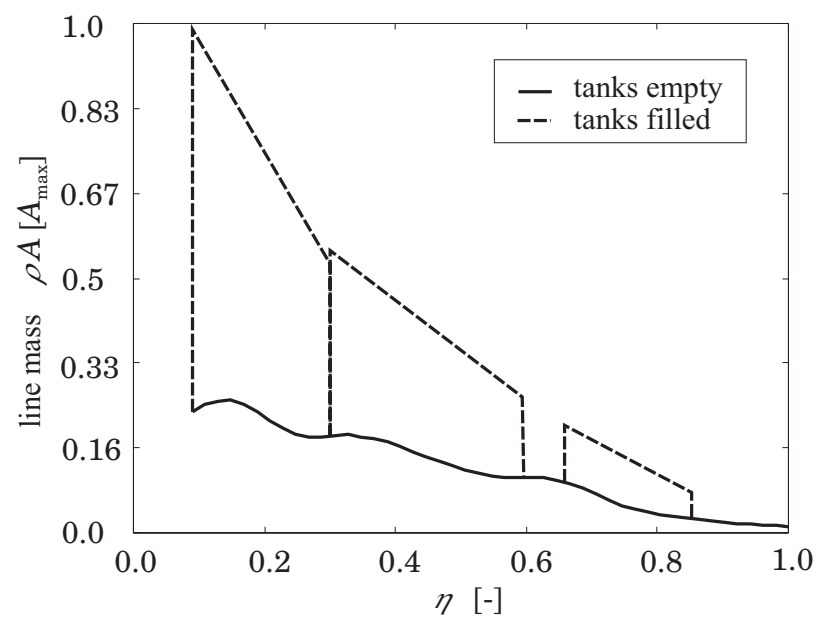

FIG 6: Spanwise mass distribution

trix equation

$$
\begin{array}{r}
\boldsymbol{M} \ddot{\mathbf{z}}+\left(\boldsymbol{D}+\frac{q}{v_{\mathrm{TAS}}} \boldsymbol{D}_{A}\right) \dot{\mathbf{z}}+\left(\boldsymbol{K}+q \boldsymbol{K}_{A}\right) \boldsymbol{z}=\cdots \\
\cdots=q \boldsymbol{Q}_{\mathbf{u}} \mathbf{u}+\boldsymbol{Q}_{R}(q) \mathbf{z}_{R}+\boldsymbol{Q}_{E} \boldsymbol{T}_{E}
\end{array}
$$

with $\boldsymbol{z}_{R}$ representing the rigid aircraft motion and $\boldsymbol{T}_{E}$ the accumulated engine thrust. The equations of motion for multi element structures are obtained by applying energy methods and the Euler-Lagrange formula (Gojny 2001).

\subsection{Control Systems}

The aeroelastic wing model includes three control surfaces: inboard aileron, outboard aileron and the outboard spoiler no. 6. Since the ailerons are primary flight control surfaces, the associated actuation system is assumed to perform high reliability and dynamic performance. Based on robust state control and estimation techniques, high bandwidth and improved damping characteristics are achieved, hence they are well suited for use in active vibration control of the wing (Gojny and Kliffken 1999). As (Carl 1988) proposes also the employment of the outer spoiler for a Load Alleviation Function (LAF) on Airbus A320, spoiler no. 6 is taken in consideration for active control, additionally. The corresponding dynamic representatives are based on physically motivated models, verified with measured frequency responses of real implemented actuation systems (Gojny 2001).

\subsection{Total Aeroservoelastic System}

Finally, the coupled aeroservoelastic model comprises a finite element wing model, aerodynamics with constant feedthrough characteristic and three closed loop actuation system models. Deploying state space model conversion, the state vector and the input vector reads

$$
\begin{aligned}
& \text { (19) } \boldsymbol{x}=\left[\begin{array}{ll|lll}
\boldsymbol{z} & \dot{\boldsymbol{z}} & \boldsymbol{w}^{\text {inb }} & \boldsymbol{w}^{\text {out }} & \boldsymbol{w}^{\mathrm{sp} 6}
\end{array}\right]^{\mathrm{T}}, \\
& \text { (20) } \boldsymbol{u}=\left[\begin{array}{lll}
\delta_{c}^{\text {inb }} & \delta_{c}^{\text {out }} & \delta_{c}^{\mathrm{sp} 6}
\end{array}\right]^{\mathrm{T}} \text {, }
\end{aligned}
$$

with $\boldsymbol{w}$ represents the closed loop actuation system states and $\boldsymbol{u}$ the command input of every active actuator and 


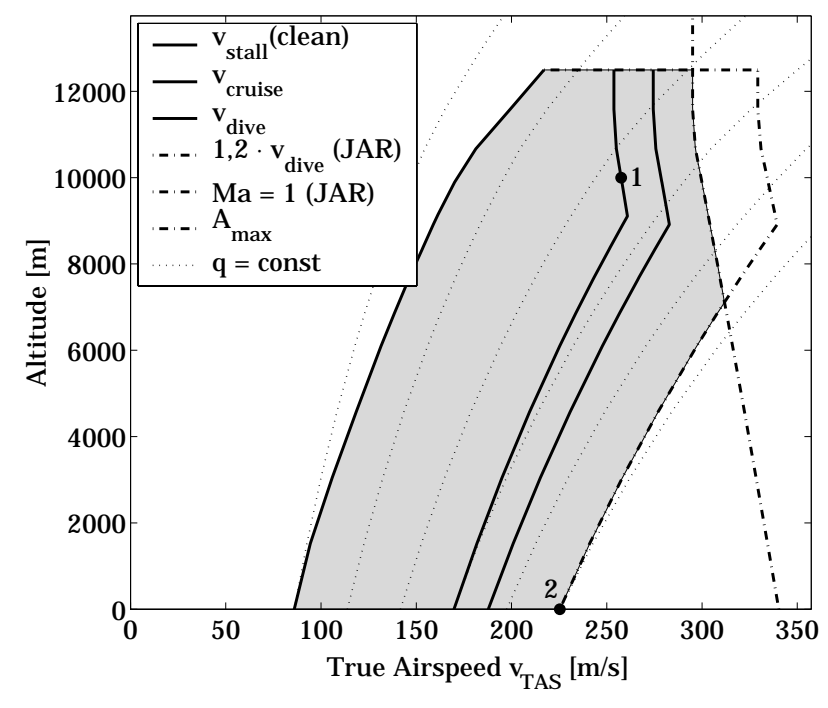

FIG 7: Generic flight envelope

the spoiler actuation system, respectively. The output vector $\mathbf{y}$ depends on the selected sensor allocation (Hanel 2001, Klett 2002). For this approach, complete observability is assumed which grants the availability of necessary state information: vertical displacement and rate as well as torsion angle and rate, respectively.

Representatives and Operating Points. During a flight mission the parameters of the aeroservoelastic system vary considerably. Regarding the flight envelope for a large transport aircraft, two characteristic operating points are selected. The corresponding parameter set and state space models denote the nominal (OP1) and critical (OP2) representative (FIG 7). As OP1 tags the ordinary cruise condition, OP2 denotes the highest dynamic pressure covered by an extended envelope. Naturally, this flight condition is not enclosed by the aircraft's flightenvelope, but structural stability must be shown for an extended envelope according to the Joint Airworthiness Requirements JAR (Joint Aviation Authorities - JAA 1997).

Scrutinising the parameter dependencies of every subsystem leads to two significant influences. The entire aeroservoelastic system is severely effected by the uncertain parameters:

- airspeed $v_{\text {TAS }}$ and altitude or the dynamic pressure $q$, respectively,

- mass $\boldsymbol{M}$, mainly effected by the varying fuel mass $M_{\text {fuel }}$.

Consequently, defining the uncertainty vector (Ackermann et al. 1993)

(21) $\quad \boldsymbol{q}=\left[\begin{array}{lll}v_{\mathrm{TAS}} & q & \boldsymbol{M}_{\mathrm{fuel}}\end{array}\right]^{\mathrm{T}}$

yields the uncertain multi-model system (1). Considering the fueling configuration, a fueling vector $\boldsymbol{f}$ is defined, which addresses the relative filling level in every tank (FIG 6)

$$
\begin{aligned}
\boldsymbol{f} & =\left[\begin{array}{lll}
f_{\text {inb }} & f_{\text {mid }} & f_{\text {out }}
\end{array}\right], \\
\boldsymbol{f}_{1} & =\left[\begin{array}{lll}
100 & 100 & 0
\end{array}\right], \\
\boldsymbol{f}_{2} & =\left[\begin{array}{lll}
0 & 100 & 100
\end{array}\right] .
\end{aligned}
$$

The left entry corresponds to the innermost wing tank. Since the tanks are usually emptied beginning with the outermost tank, the first configuration $\boldsymbol{f}_{1}$ always occurs during a normal flight mission, whereas $\boldsymbol{f}_{2}$ may only occur due to a malfunction in the fuel management system. Preceding investigations reveal the latter to be most critical.

\section{MODE CONTROL APPLICATION}

Improving modal damping characteristics of an uncertain MIMO plant family requires a robust control law. Due to pragmatic realisation aspects, vibration control is aspired to be realised as a low order compensator. Unfortunately, each increase in order results in additional closed-loop eigenvalues that must be robustly stabilised, too (Ackermann et al. 1993). Therefore, the subsequent design focusses on static output feedback $\boldsymbol{R}$, whereas the outputs represent $m$ acceleration measurements at predefined locations all over the wing. The commands of the closed loop actuation systems $\boldsymbol{u}$ consist of the flight mechanically commanded deflections by pilot or auto pilot and the weighed output feedback $-\boldsymbol{R} \boldsymbol{y}$ to augment the structural damping actively. The current multi-model system comprises four representatives in state-space form, each individual is determined by different entries of the uncertainty vector $\boldsymbol{q}(21)$.

Plant and constraints. Every aeroservoelastic representative totally consists of $n=376+20=396$ states (aeroelastic subsystem and closed loop PFC actuation systems) and $p=3$ inputs (inboard aileron, outboard aileron and outer spoiler). From preceding investigations result a considerable dominance of the first four low-frequency eigen-

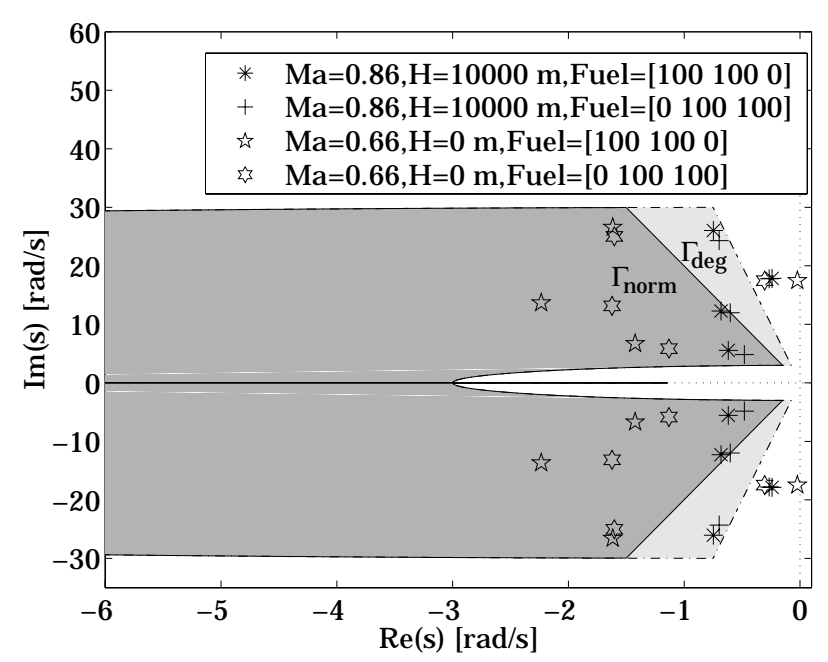

FIG 8: Open loop eigenvalues 
modes (Gojny 2001, Klett 2002). Considering the open loop dynamics in detail, the conjugate complex eigenvalues associated to the third mode (FIG 4) reveal a quite weak damping. The critical root loci display the damping ratio $d \in[0.0013,0.0017]$ for the entire multi-model set (FIG 8). The entire set of dominant aeroelastic frequencies are well inclosed within the interval of the controls. By means of robust state control (Gojny and Kliffken 1999), the extended bandwidth of the aileron actuation systems amounts to e.g. $f_{B} \approx 12 \mathrm{~Hz}$. Hence, the actuation systems are capable of operating within a mode control function. Furthermore, a dual approach is taken into consideration: Applying the structural performance index to the total model and optionally, performing model order reduction in advance plus state controller design afterwards.

Requirements. Regarding the availability of control surfaces and actuation systems, the controller design differentiates between normal and degraded condition. In normal condition all three control surfaces are employed to grant the specified nominal damping ratio, i.e. to achieve the eigenvalue region $\Gamma_{\text {norm }}$. Assuming a double-failure of one actuation system leads to degraded conditions. The minimum damping ratio of the controlled system has to amount at least to a significant residue, say to the half compared to normal condition. Incorporating the outlined scenario yields an additional design constraint, stated by the eigenvalue region $\Gamma_{\text {deg }}$ (FIG 8). Considering the worst case, e.g. only the inboard or outboard aileron is available for the structural damping augmentation, the feedback matrix structurally yields, according to (20)

$$
\tilde{\boldsymbol{R}} \in\left\{\left[\begin{array}{ccc}
* & \cdots & * \\
0 & \cdots & 0 \\
0 & \cdots & 0
\end{array}\right],\left[\begin{array}{ccc}
0 & \cdots & 0 \\
* & \cdots & * \\
0 & \cdots & 0
\end{array}\right]\right\}
$$

Wherein $*$ represents arbitrary entries (Lunze 1997). According to (12), the adjusted robustness criterion valuates the root loci with respect to $\Gamma_{\mathrm{deg}}$ for degraded condition

$$
J_{D}=\sum_{j=1}^{p-1} J_{R}\left(\lambda_{R i}\left(\tilde{\boldsymbol{R}}_{j}, \Gamma_{\mathrm{deg}}\right)\right), i=1 \ldots n
$$

Prepending the design objective should be determined quite moderate, the minimum damping specification of the controlled system is set to at least $d_{\text {min }}=0.05$ regarding normal condition and $d_{\min }=0.025$ for degraded condition, respectively. The maximum bandwidth $\omega_{\max } \approx \omega_{B} / 2$ results from minimum phase shift requirements of the actuation systems and beyond a minimum frequency shift of the aeroelastic eigenvalues (FIG 8). Moreover, this selection ensures a sufficient performance margin even for degraded actuation system dynamics, e.g. a slight internal leakage etc.. The dashed line denotes the edge of $\Gamma_{\mathrm{deg}}$.

Optimisation. Fundamentally, the outlined approach bases on standard implementation, e.g. solver from Matlab Optimisation Toolbox for constrained performance index optimisation. The engineer only has to define the weighted total performance index

(25) $\boldsymbol{J}=\boldsymbol{U}\left[\begin{array}{lllll}J_{R} & J_{D} & J_{N} & J_{S} & \cdots\end{array}\right]^{\mathrm{T}}$.

and corresponding bounds with respect to the design variables (11). Wherein $\boldsymbol{U}$ either represents a diagonal weighing matrix for performance vector optimisation or a vector in case of optimising a scalar performance index, optionally.

Closed loop dynamics. The MIMO controller candidate $\boldsymbol{R}$ must demonstrate its performance with reference to the total aeroservoelastic model $(n=396)$. Despite the dual design approach (order reduction and state controller design versus direct design), both operate as output feedback (order $n=8$ ) relying on sensor positioning strategies and spillover filtering (Choi and Park 2000). Since one actuation system model is replaced by a real Airbus A330/340 inboard aileron actuation system with state control is incorporated, the verification is performed as realtime hardware-in-the-loop simulation (Gojny 2001).

Assuming nominal operating conditions, the eigenvalue analysis confirms the static output controller meeting the damping augmentation requirement (FIG 9). The desired pole region $\Gamma_{\text {norm }}$ contains the entire closed loop eigenvalue set in consideration of the smallest reachable feedback gains (25). Remarkable effects can be stated at the poles which are associated to the critical third and the fourth mode, whereas the remainder of the eigenvalues substantially remain unaffected.

FIG 10 displays the step response of the wing tip to an abrupt change in the angle of attack $\alpha_{0}(t)=1^{\circ} \sigma(t)$. The figure is subdivided into four parts, the system response in bending and twist, i.e. vertical displacement and torsion considering the wing tip section as well as the surface deflection of the inboard and outboard aileron, exemplarily. The latter illustrates the necessary

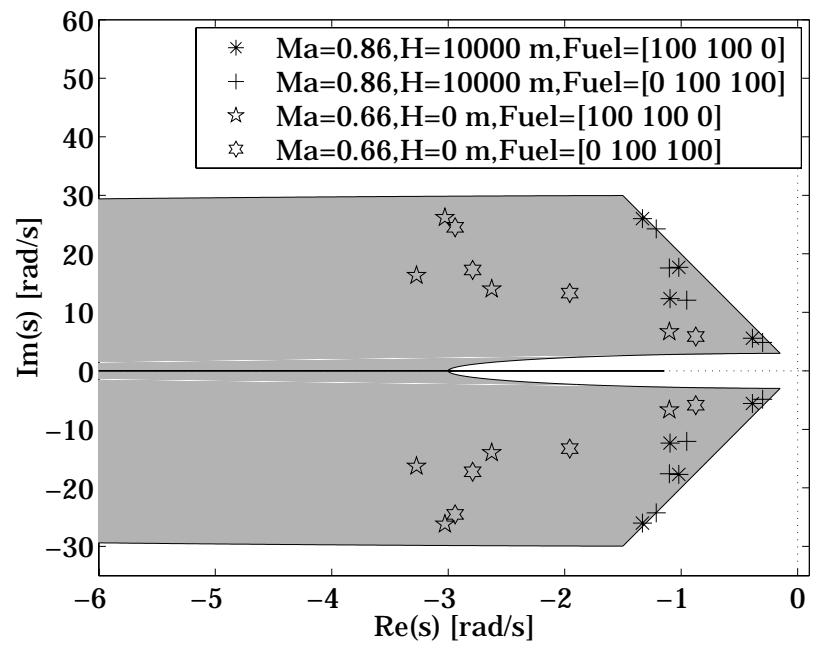

FIG 9: Closed loop eigenvalues 

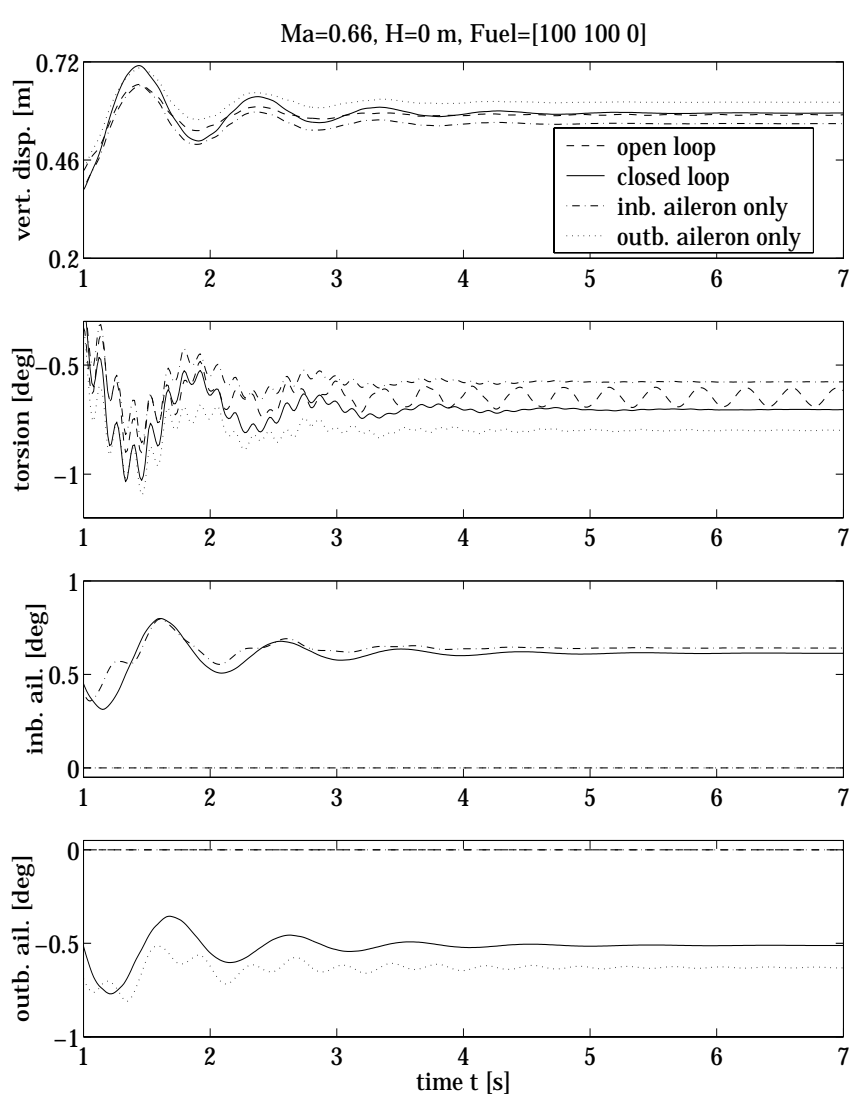

FIG 10: $\alpha_{0}$ step response

amplitudes for compensation due to $\boldsymbol{R}$. The open loop signals, which means no active damping augmentation is present, represent the basis for comparison purposes. Remarkably, the weakly damped third mode (FIG 8) proves its dominance, especially regarding the twist curve and assists not only to vibration stress which finally leads to structural fatigue failures, but significantly affects the system stability. The feedback controller improves the closed loop damping characteristic within a retiring interval of $T_{5 \%} \approx 3 \mathrm{~s}$ as expected. Beyond, the MIMO controller also demonstrates its capability to ensure full functionality in a degraded condition, even if only one aileron actuation system is available. Whereas in normal operation both $\delta^{\text {inb }}$ and $\delta^{\text {out }}$ evince a high ratio of bending compensation, in degraded condition that only holds for the inboard aileron in stand alone operation. Obviously, the outboard aileron controller lane is moderately stronger influenced by torsional vibration forms. Comparing the normal and degraded step responses to the open loop dynamics reveals no significant deterioration of performance, which implies a slight effect of the outer spoiler only. With respect to the current example the spoiler may be interpreted as auxiliary device only, but for further application and functionality enhancements it holds promising prospects.

Altogether, based on the outlined enhanced eigenstructure assignment technique, the static MIMO feedback ensures vibration control even for degraded system configurations. Generically, the closed loop system is also qualified by ro- bustness. With regard to the flexible wing, the outlined design is performed considering normal and degraded performance from the outset. Moreover, the proposed procedure denotes modularity concerning the integration of performance specifications and keeps physical insight, e.g. with reference to the dynamic specifications.

\section{CONCLUDING REMARKS}

Eigenstructure assignment represents a state of the art controller design method considering the discipline of flight control. Engineers gained much experience in application, managing specifications like damping, rise time bandwidth and decoupling. Unfortunately, the eigenspace specification meant a serious disadvantage and stated inapplicability with respect to vibration control tasks. Conserving familiar engineering parameters and insight even in vibration controller design succeeds in introducing significant modifications to eigenstructure assignment. The controller synthesis itself is based on Roppenecker's formula (Roppenecker 1990). By means of multiobjective optimisation, even potentially competing objective specifications are integrated within one approach,

- time domain specifications, e.g. settling time,

- frequency domain specifications, e.g. cut-off frequency,

- dynamic input-output requirements, quadratic i/o criterion with linear constraints (LQ), dynamic uncertainties,

- parametric requirements, e.g. minimum gain, uncertainty bounds,

- restrictions due to the system architecture, e.g. quantity and location of sensors, actuators, etc..

The application had been demonstrated successfully relating to an uncertain aeroservoelastic multiple-input multiple-output plant. An infinitive set of flight conditions and fuel configurations of a flexible wing structure confirms the need for robust control. The set of representatives was composed of linear multivariable multi-model systems with the system inputs, inboard and outboard aileron and the outer spoiler. Emphasis must be placed on quite moderate design objectives of the active damping augmentation function, according to the second basic rule of robust control (Ackermann et al. 1993). Additionally, the proposed design procedure enables to define different dynamic characteristics, simultaneously, which was exemplified by normal and degraded specification. But could also be utilised to define e.g. flightmechanical and aeroelastic pole regions for separated robust stabilisation. In descending order, the total performance index resulted as a weighted combination of the robustness criterion, degraded performance criterion, controller norm criterion and structural criterion. The suitability of the resulting low-order static output controller had been verified for the normal and degraded configuration. The performance of the controlled full scale aeroservoelastic system had 
been demonstrated by real time simulation covering a real PFC actuation system hardware-in-the-loop. Investigations with respect to structural displacement revealed a significant damping augmentation regarding external disturbances as well as self excited vibrations in operative and even in the degraded system condition.

Active damping augmentation by primary flight control surfaces could efficiently effect the weakly damped aeroelastic wing motions, the proposed controller design method provides promising properties for further challenging applications. Recent activities address the integration of a multi-model system of a complete flexible commercial aircraft into the design and real-time simulation environment.

\section{REFERENCES}

Ackermann, J. et al.: 1993, Robuste Regelung - Analyse und Entwurf von linearen Regelungssystemen mit unsicheren physikalischen Parametern, Springer, Berlin, Germany.

Boyd, S.P. and Barrat, C.H.: 1991, Linear Controller Design — Limits of Performance, Prentice-Hall, Englewood Cliffs NJ, USA.

Carl, U.B.: 1988, Elektrische Primärsteuerung (fly by wire): Flugsteuerungssysteme neuer Technologie, Technical report, Messerschmidt-BölkowBlohm GmbH, Ziviles Komponenten Programm (ZKP), Bremen, Germany. UT 122-88.

Chiappa, C. et al.: 1998, Flexible structure control by modal multi-model approach: Application to a flexible aircraft, International Council of Aeronautical Sciences, $21^{\text {st }}$ ICAS Congress, Melbourne, Australia. (A98-31461).

Choi, J.W. and Park, U.S.: 2000, Spillover suppression in a flexible structure using eigenstructure assignment, Guidance, Navigation and Control Conference and Exhibit, Denver CO, USA. (AIAA2000-3953).

Dardenne, I.: 1998, Développement de Méthologies pour la Synthèse de Lois de Commande d'un Avion Transport Souple, PhD thesis, No. 256, L'École Nationale Supérieure de l'Aéronautique et de l'Espace (SUPAERO), Toulouse, France.

Dowell, E.H. (ed.): 1995, A Modern Course in Aeroelasticity, 3. edn, Kluwer, Dordrecht, The Netherlands.

Föllinger, O.: 1994, Regelungstechnik, 8. edn, Hüthig, Heidelberg, Germany.

Gojny, M.H. and Kliffken, M.G.: 1999, Numerisch robuste Implementierung von Abtastreglern für Flugsteuerungsaktuatoren, atAutomatisierungstechnik 47, 414-420.

Gojny, M.H. and Carl, U.B.: 2001, Robust mode control of a flexible wing structure, International Conference on Recent Advances in Aerospace Aerospace Actuation Systems and Components, Toulouse, France, pp. 91-98.
Hanel, M.: 2001, Robust Integrated Flight and Aeroelastic Control System Design for a Large Transport Aircraft, PhD thesis, Universität Stuttgart. Fortschritt-Berichte VDI Series 8 No. 866, VDIVerlag, Dusseldorf, Germany.

Klett, L.: 2002, Optimierte Sensorpositionierung zur aktiven Dämpfung eines aeroservoelastischen Tragflügels, Master's thesis, Technical University of Hamburg-Harburg, Germany.

Kubica, F.: 1995, Conception de Lois de Commande pour un Avion Souple, PhD thesis, No. 178, L'École Nationale Supérieure de l'Aéronautique et de l'Espace (SUPAERO), Toulouse, France.

Kubica, F. and Livet, T.: 1994, Flight control law synthesis for a flexible aircraft, AIAA Guidance, Navigation and Control Conference, Phoenix AZ, USA, pp. 775-783. (AIAA-94-3630).

Lind, R. and Brenner, M.: 1999, Robust Aeroservoelastic Stability Analysis, Springer, London, England.

Livet, T.: 1995, Conception de Lois de Commande Robustes et Performantes pour le Pilotage Automatique d'Avions Civile: Application au Cas de l'Avion Souple, PhD thesis, No. 174, L'École Nationale Supérieure de l'Aéronautique et de l'Espace (SUPAERO), Toulouse, France.

Lunze, J.: 1997, Regelungstechnik2, Springer, Berlin, Germany.

MacKinnon, A. and Stollery, J.L.: 1993, Variable camber wing, Technical report, Cranfield University, Cranfield, England. COA Rep. 9304.

Mukhopadhyay, V. et al.: 2001, Benchmark active control technology, Journal of Guidance Control and Dynamics 24(1), 146-185. Part III.

Joint Aviation Authorities - JAA (ed.): 1997, Joint Aviation Requirements, JAR-25: Large Aeroplanes, Westwood Digital Limited, Cheltonham, England.

Roppenecker, G.: 1990, Zeitbereichsentwurf linearer Regelungen, Oldenbourg, Munich, Germany.

Schuler, J.: 1998, Flugregelung und aktive Schwingungsdämpfung für flexible Grossraumflugzeuge, $\mathrm{PhD}$ thesis, University of Stuttgart. FortschrittBerichte VDI No. 688 Series 8, VDI-Verlag, Dusseldorf, Germany.

Seyffarth, K.: 1993, Comfort in turbulence for a large civil transport aircraft, International Forum on Aeroelasticity and Structural Dynamics, Strassbourg, France.

She, T.: 1992, Active Vibration Control by Eigenstructure Assignment, $\mathrm{PhD}$ thesis, Katholieke Universiteit Leuven, Leuven, Belgium.

Wu, C.H.: 1999, Flight Dynamics of a Flexible Aircraft, PhD thesis, Technical University of Brunswick, Shaker Verlag, Aachen, Germany. ZLRForschungsbericht 99-02. 


\section{NOTATIONS AND ABBREVIATIONS}

Variables and parameters.

\begin{tabular}{|c|c|c|}
\hline$\alpha$ & {$\left[{ }^{\circ}\right]$} & dynamic angle of attack \\
\hline $\boldsymbol{A}$ & {$[-]$} & system matrix \\
\hline $\boldsymbol{B}$ & {$[-]$} & input matrix \\
\hline$\gamma$ & {$[-]$} & scalar penalty \\
\hline$c$ & {$[-]$} & aerodynamic derivative \\
\hline$C$ & {$[-]$} & output matrix \\
\hline$\partial \Gamma$ & {$[-]$} & stability boundary \\
\hline$\delta$ & {$\left[{ }^{\circ}\right]$} & control surface deflection \\
\hline $\boldsymbol{D}$ & {$[-]$} & feedthrough matrix \\
\hline $\boldsymbol{D}$ & {$[-]$} & damping matrix \\
\hline$d$ & {$[-]$} & scalar damping ratio \\
\hline$f$ & {$[\mathrm{~Hz}]$} & frequency \\
\hline $\boldsymbol{f}$ & {$[-]$} & relative fueling vector \\
\hline$\Gamma$ & {$[-]$} & stability region \\
\hline$\eta$ & {$[\mathrm{m}]$} & wing span coordinate \\
\hline$h$ & {$[\mathrm{~m}]$} & altitude \\
\hline$J$ & {$[-]$} & performance index \\
\hline $\boldsymbol{K}$ & {$\left[\mathrm{kg} / \mathrm{s}^{2}\right]$} & stiffness matrix \\
\hline$\lambda$ & {$[\mathrm{rad} / \mathrm{s}]$} & eigenvalue \\
\hline$l$ & {$[-]$} & number of system representatives \\
\hline$L$ & {$[-]$} & design variable \\
\hline $\boldsymbol{L}$ & {$[-]$} & matrix of design variables \\
\hline$m$ & {$[-]$} & number of outputs \\
\hline$M$ & {$[\mathrm{~kg}]$} & mass matrix \\
\hline$M a$ & {$[-]$} & Mach number \\
\hline$n$ & {$[-]$} & system order \\
\hline$\omega$ & {$[\mathrm{rad} / \mathrm{s}]$} & radian frequency \\
\hline$p$ & {$[-]$} & number of inputs \\
\hline $\boldsymbol{p}$ & {$[-]$} & parameter vector \\
\hline $\boldsymbol{P}$ & {$[-]$} & matrix of parameter vectors \\
\hline$Q$ & {$[-]$} & input matrix \\
\hline$q$ & {$[-]$} & vector of uncertain parameters \\
\hline
\end{tabular}

$q \quad[\mathrm{~Pa}]$

dynamic angle of attack

scalar penalty

erodynamic derivative

feedthrough matrix

damping matrix

mping ratio

stability region

altitude

erformance index

ness matrix

number of system representatives

design variable

dynamic pressure

air density

normalised fueling scale factor

feedback gain

feedback matrix

unity step function

complex frequency $s=\delta \pm \mathrm{j} \omega$

angular displacement at $\eta$

time quantity

time interval

thrust matrix

input vector

performance weighing matrix

scalar input

air speed

eigenvector

generalised actuation system states

state vector

output vector

vertical displacement

vector of physical displacement
Indexes.

(Note: The listed symbols may be used as lower as well as upper index.)

$\begin{array}{lll}- & {[-]} & \text { lower boundary } \\ + & {[-]} & \text { upper boundary } \\ \text { max } & {[-]} & \text { maximum } \\ \text { min } & {[-]} & \text { minimum } \\ 0 & {[-]} & \text { initial } \\ 5 \% & {[-]} & \text { settling time } \\ \alpha & {[-]} & \text { angle of attack } \\ A & {[-]} & \text { aerodynamic } \\ c & {[-]} & \text { command } \\ \delta & {[-]} & \text { control surface } \\ \text { deg } & {[-]} & \text { degraded condition } \\ D & {[-]} & \text { dive } \\ d & {[-]} & \text { disturbance } \\ e & {[-]} & \text { example } \\ E & {[-]} & \text { engine } \\ \text { inb } & {[-]} & \text { inboard } \\ l & {[-]} & \text { lift } \\ l & {[-]} & \text { local wing coordinates } \\ m & {[-]} & \text { momentum } \\ \text { mid } & {[-]} & \text { mid } \\ \text { norm } & {[-]} & \text { normal condition } \\ N & {[-]} & \text { norm-based } \\ \text { out } & {[-]} & \text { outboard } \\ R & {[-]} & \text { closed loop, robust } \\ R & {[-]} & \text { rigid } \\ S & {[-]} & \text { structural } \\ S & {[-]} & \text { local aileron coordinates } \\ \text { SP6 } & {[-]} & \text { outer spoiler } \\ u & {[-]} & \text { input } \\ z & {[-]} & \text { vertical displacement }\end{array}$

\begin{tabular}{|c|c|c|}
\hline \multicolumn{3}{|c|}{ Abbreviations. } \\
\hline ACT & {$[-]$} & active control technology \\
\hline EAS & {$[-]$} & equivalent air speed \\
\hline $\mathrm{I} / \mathrm{O}$ & [-] & input-output \\
\hline LAF & {$[-]$} & load alleviation function \\
\hline LQ & {$[-]$} & $\begin{array}{l}\text { quadratic performance function with } \\
\text { linear constraints }\end{array}$ \\
\hline LQG & {$[-]$} & linear quadratic gaussian \\
\hline PFC & {$[-]$} & primary flight control \\
\hline MIMO & {$[-]$} & multiple input multiple output \\
\hline SQP & {$[-]$} & sequential quadratic programming \\
\hline TAS & {$[-]$} & true air speed \\
\hline
\end{tabular}

\section{ACKNOWLEDGEMENT}

The authors thank the German Federal Ministry of Economics and Technology for promoting the current investigations within the framework of the german aeronautical research programme and Airbus Deutschland $\mathrm{GmbH}$ for supporting the research project Systemtechnische Untersuchungen zur Strukturregelung flexibler Flugzeuge SYSFF. 